\title{
Air Quality Risk Index (AQRI) and its application for a megacity
}

\author{
A. Ahmadi ${ }^{1} \cdot$ M. Abbaspour ${ }^{1,2} \cdot$ R. Arjmandi $^{1} \cdot$ Z. Abedi ${ }^{1}$
}

Received: 6 April 2015/Revised: 1 June 2015/Accepted: 21 June 2015/Published online: 7 August 2015

(C) Islamic Azad University (IAU) 2015

\begin{abstract}
Urban air quality is a major concern throughout the world. The concentration of human activities in a relatively small area puts enormous pressure on urban systems and has led to numerous environmental problems which have created major problems for urban air quality management. The complex nature of air pollution, especially with respect to health impacts in urban areas, has prompted attempts to define the so-called indicators that condense and simplify the available monitoring data to make them suitable for public reporting and decision makers. Several concepts and indicators exist to measure and rank urban areas in terms of their infrastructural, socioeconomic and environment-related parameters. In past four decades different indices have been introduced, such as Pollutant Standards Index, Air Quality Index (AQI), Air Quality Health Index and Aggregate Risk Index, but none of them have considered importance of the integrated health, environment and safety factors relevant to the extended risk factors involved, such as diverse economic losses and negative socioeconomic aspects. Therefore an integrated AQI, so-called, Air Quality Risk Index which considers environmental conditions, and economic losses involved were applied for the city of Tehran as a megacity. The results indicated that this index can be applied to define the actual condition(s) of urban air quality and to achieve sustainability and resiliency.
\end{abstract}

A. Ahmadi

ahmadyaida@yahoo.com

1 Department of Environmental Management, Science and Research Branch, Islamic Azad University, Tehran, Iran

2 School of Mechanical Engineering, Sharif University of Technology, Tehran, Iran
Keywords Air pollution · Air Quality Index · Air Quality Health Index · Air Quality Risk Index · Health, safety and environment

\section{Introduction}

Air pollution occurs when the air contains gases, dust, fumes or odor in harmful amounts, that is, amounts that could be harmful to the health or comfort of humans and animals or which could cause damage to plants and materials (Gurjar et al. 2012).

Major sources of air pollution in urban areas are stationary sources such as industrial plants and mobile sources mainly in the transportation sector, which uses fossil fuels as the source of energy (World Health Organization 2010). High smokestacks from industrial sources, such as refineries and coal-fired electrical power generating plants, can carry pollutants such as acidic aerosols and sulfur oxides over hundreds of kilometers by air that moves freely across international borders (Environmental Protection Agency 2009).

Urban air quality is a major concern throughout the world (World Health Organization 2014). This, in fact, is an immense issue with diverse effects on earth environments in different parts of the world or even within a specific region.

Air pollution is a major worldwide concern in an increasing number of megacities around the world (Prinn et al. 2005). Generally speaking, all mitigation plans by governments are aiming toward execution of programs which can ultimately result in the reduction in pollutant's concentrations. In order to deal with the complex nature of air pollution, especially with respect to health, has been a motive to define the so-called indicators which simplify the 
collected monitoring data and to make them suitable for reporting to the public and decision making by authorities (Gurjar et al. 2012).

The Pollutant Standards Index (PSI) was introduced in the mid-1970s to provide a uniform system of measuring pollution levels for the major air pollutants. The PSI was used in a number of countries, including the USA and Singapore; however, in early 1980s, the US EPA replaced the PSI with a new index, so-called Air Quality Index (AQI), which pays more attention to air quality. AQI measures and reports on six key urban air pollutants: ozone $\left(\mathrm{O}_{3}\right)$; particulate matter $\left(\mathrm{PM}_{10}, \mathrm{PM}_{2.5}\right)$; carbon monoxide (CO); nitrogen dioxide $\left(\mathrm{NO}_{2}\right)$; and sulfur dioxide $\left(\mathrm{SO}_{2}\right)$ (Ontario Ministry of the Environment 2005; Ahadi et al. 2013).

These pollutants have long been associated with human adverse health and environmental effects at elevated levels. Hourly readings for each pollutant at various stations are assigned an AQI value from zero upward using a common scale (Ontario Ministry of the Environment 2006). EPA calculates the AQI for major air pollutants regulated by the Clean Air Act (Environmental Protection Agency 2009). Each category has a descriptive name reflecting levels of health concern (ranging from good through very hazardous), an associated color, and an advisory statement (Committee on Environmental Health 2004). The higher the AQI value, the greater the level of air pollution, and the greater the health concern. AQI values at or below 100 are generally thought of as satisfactory, relations and features of AQI are presented in US EPA, Kansas Department of Health and Environment Bureau (Environmental Protection Agency 2009; U.S. Environmental Protection Agency Of Standards 2006; Kansas Department of Health and Environment Bureau 2005).

To focus more on the health factor in air pollution indices, a new index called Air Quality Health Index (AQHI) was introduced in early 2000 to describe the connection between the general "health situation" and ambient air pollution in urban areas (Development Status Report of the Commissioner of the Environment 2009).

The AQHI was applied in 10 urban areas of Canada. It measured on a color-coded scale from 1 to $10+$ (Chen and Copes 2013). The higher the number, the greater the health risk associated with local air quality. The AQHI numeric ratings (from 1 to $10+$ ) are grouped into risk categories (low, moderate, high, very high) that are designed to identify the level of health risk in a "quick and easy" manner. The AQHI is a tool that could be used by people to reduce their short-term exposure to air pollution and plan, on a daily basis, to modify their behavior and reduce their personal health risk. Therefore, the AQHI responds to the acute, or short-term, changing levels of health risk associated with air pollution. Each category is associated with specific health advice for those at risk (children, seniors, people with heart or lung conditions and diabetics), and the general population. The relations and features of AQHI are presented in Hasselback, Health Canada and Environment, and Hindy and Pennell (Hasselback 2010; Health Canada and Environment 2009; Hindy and Pennell 2010; Health Protection Agency for the Committee on the Medical Effects of Air Pollutants 2011).

During the recent years, some other indices such as Aggregate Risk Index (ARI) (Sicard et al. 2012) have been introduced. However, none of these indices has reflected safety factors and diverse economic impacts due to the problem of air pollution. Therefore, an integrated AQI, called, Air Quality Risk Index (AQRI) has been introduced which not only considers environmental conditions and health issues but also brings economic losses involved into account. Moreover, a case study has been carried out with the available data of the year 2010 which was used to evaluate the state of Tehran megacity AQRI.

\section{Materials and methods}

Over the past four decades, as stated, different indices have been introduced for air quality, but none of them have paid attention to the three factors of health $\left(R_{\mathrm{H}}\right)$, safety $\left(R_{\mathrm{S}}\right)$ and environment $\left(R_{\mathrm{E}}\right)$ simultaneously.

The diverse consequences of air pollution are not limited only to the health and environment, but air pollution also imposes direct and indirect expenses on a community. For example, it can damage building's facade, increase accidents due to reduced visibility, cause the closure of the government offices, educational institutions and increase the hospital admissions due to respiratory diseases, which impose economic losses and external costs on metropolitan economy. This was a reason to bring the safety (damage and loss) $\left(R_{\mathrm{S}}\right)$ item into the definition of AQI. Therefore, AQRI can be used as an index to control air quality, health conditions and economic losses due to air pollution. As a result, AQRI can be used as an urban air quality management tool for policy makers.

The National Health Service (NHS) of the UK published a report in 2007/2008 stating that the hospital admissions triggered by air pollution have imposed additional costs to the country. For example, in 2007/2008, there were over 74,000 emergency admissions to hospitals because of asthma, and the NHS's non-elective spell tariff was $£ 612$ million for that year. The same report has indicated that air pollution has put considerable expenses on the shoulders of communities. These expenses have cost the tax payers $£ 8.5-£ 20.2$ billion in the year 2005. The Air Quality Management Resource Centre of the UK has noted that the health impacts of air quality in the UK are almost twice 
that of physical inactivity, estimated to be $£ 10.7$ billion per annum (House of Commons Environmental Audit Committee 2010). Air pollution has adverse short-term effects on health (Katsouyanni et al. 1996) and bears major impact on public health especially on cardiovascular problems (Kassomenos et al. 2008; Sunyera et al. 2003). Clear links between asthma and other respiratory diseases and air quality indicate that health risks $\left(R_{\mathrm{H}}\right)$ are also another important factor to be considered in AQRI (House of Commons Environmental Audit Committee 2010).

Air pollution has a wide range of environmental impacts, including loss of biodiversity, reduction in crop yields and contribution to climate change. At the present time, $\mathrm{NO}_{\mathrm{x}}, \mathrm{SO}_{\mathrm{x}}, \mathrm{CO}, \mathrm{PM}_{2.5}$ and $\mathrm{PM}_{10}$ have the most sig- damages, $R_{\mathrm{S}}$, and $\mathrm{AQHI}$ as health-related risk, $R_{\mathrm{H}}$, indices can be integrated in order to define and measure AQRI. It should be noted that in calculation of AQI, the six pollutant items are used as indicated in Eq. (1) (Environmental Protection Agency 2009), while for the calculation of AQHI, only three pollutants are used as shown in Eq. (2):

$I_{\mathrm{p}}=\frac{I_{\mathrm{Hi}}-I_{\mathrm{Lo}}}{B P_{\mathrm{Hi}}-B P_{\mathrm{Lo}}}\left(C_{\mathrm{p}}-B P_{\mathrm{Lo}}\right)+I_{\mathrm{Lo}}$

$I_{\mathrm{p}}$, Air Quality Index (AQI); $C_{\mathrm{p}}$, the rounded concentration of pollutant; $B P_{\mathrm{Hi}}$, the concentration breakpoint that is $\geq$ to $C_{\mathrm{p}} ; B P_{\mathrm{Lo}}$, the concentration breakpoint that is $\leq$ to $C_{\mathrm{p}} ; I_{\mathrm{Hi}}$, the AQI value corresponding $B P_{\mathrm{Hi}} ; I_{\mathrm{Lo}}$, the AQI value corresponding $B P_{\text {Lo }}$

$\mathrm{AQHI}: \frac{10}{10.4\left(100\left(\mathrm{e}^{\left(0.000871 \times \mathrm{NO}_{2}\right)}-1+\mathrm{e}^{\left(0.000537 \times \mathrm{O}_{3}\right)}-1+\mathrm{e}^{\left(0.000487 \times \mathrm{PM}_{2.5}\right)}-1\right)\right)}$

nificant impacts on the environment. For example, excess nitrogen from emissions of ammonia and $\mathrm{NO}_{\mathrm{x}}$ leads to excessive plant growth and decay (eutrophication) that disturbs the biodiversity of both land-based and waterbased ecosystems. It also contributes to the acidification of ecosystems, which has adverse effects on natural and seminatural habitats.

Ozone has a direct effect on plants, damaging leaf structure and reducing growth, and as a result, damaging their defense mechanisms. Exceeded ozone exposures to critical loads can affect crops, forests and semi-natural vegetation over substantial areas. Ground-level ozone also contributes to global warming indirectly by reducing carbon absorbed by vegetation (U.S. Environmental Protection Agency 1997).

As previously described, critical loads of exposure to one or more air pollutants will increase the risk of damage to certain sensitive elements of the environment that are essential for survival of many species on Earth. Thus, paying attention to environmental risks $\left(R_{\mathrm{E}}\right)$ is not only important for biodiversity, but is also important for quality of life and for human activities.

Acknowledging these factors, that is, $R_{\mathrm{H}}, R_{\mathrm{S}}$ and $R_{\mathrm{E}}$, AQRI can be defined as follows.

Table 1 presents an outline of the sources, impacts, prevention and control methods for the most important air pollution factors (U.S. Environmental Protection Agency 1997, 2006).

This outline indicates that AQI as environmental risk index, $R_{\mathrm{E}}$, safety (damages and losses), as related economic
All pollutant concentrations in this algorithm are 3-h average and in terms of $\frac{\mu \mathrm{g}}{\mathrm{m}^{3}}$ (Chen and Copes 2013).

In order to evaluate the damages and losses (safety factor), $R_{\mathrm{S}}$, the amount of damages per capita due to different consequences of poor urban air quality should be evaluated. For example, for the case of a resilient city with acceptable air quality, there are no amount of damage and therefore the $R_{\mathrm{S}}$ is zero, while if the amount of damage exceeds 10 million dollars for a city with a population of 1 million, the $R_{\mathrm{S}}$ is 10 (dollars per capita/per day), which indicates a high value for $R_{\mathrm{S}}$.

\section{Calculation of AQRI}

Air Quality Risk Index (AQRI) can be evaluated using three issues of health AQHI $\left(R_{\mathrm{H}}\right)$, safety (damages and losses) $\left(R_{\mathrm{S}}\right)$ and environment AQI $\left(R_{\mathrm{E}}\right)$, which the amount of $R_{\mathrm{H}}, R_{\mathrm{S}}, R_{\mathrm{E}}$ can be evaluated from Eq. (3).

$R_{i}=\mathrm{SS}_{i} \times \mathrm{LO}_{i}$

$R$ risk; $i \mathrm{H}, \mathrm{S}, \mathrm{E} ; L$ likelihood of occurrence; $S S$ subjective severity.

Subjective severity (SS), which indicates the severity of damages considering each of the three elements, that is, AQHI, safety (damages and losses), and AQI can be evaluated from Table 2 .

Likelihood of occurrence (LO) is the probability of occurrence of a possible event in a certain time period in four levels: 1-low, 2-medium, 3-high and 4-very high. 


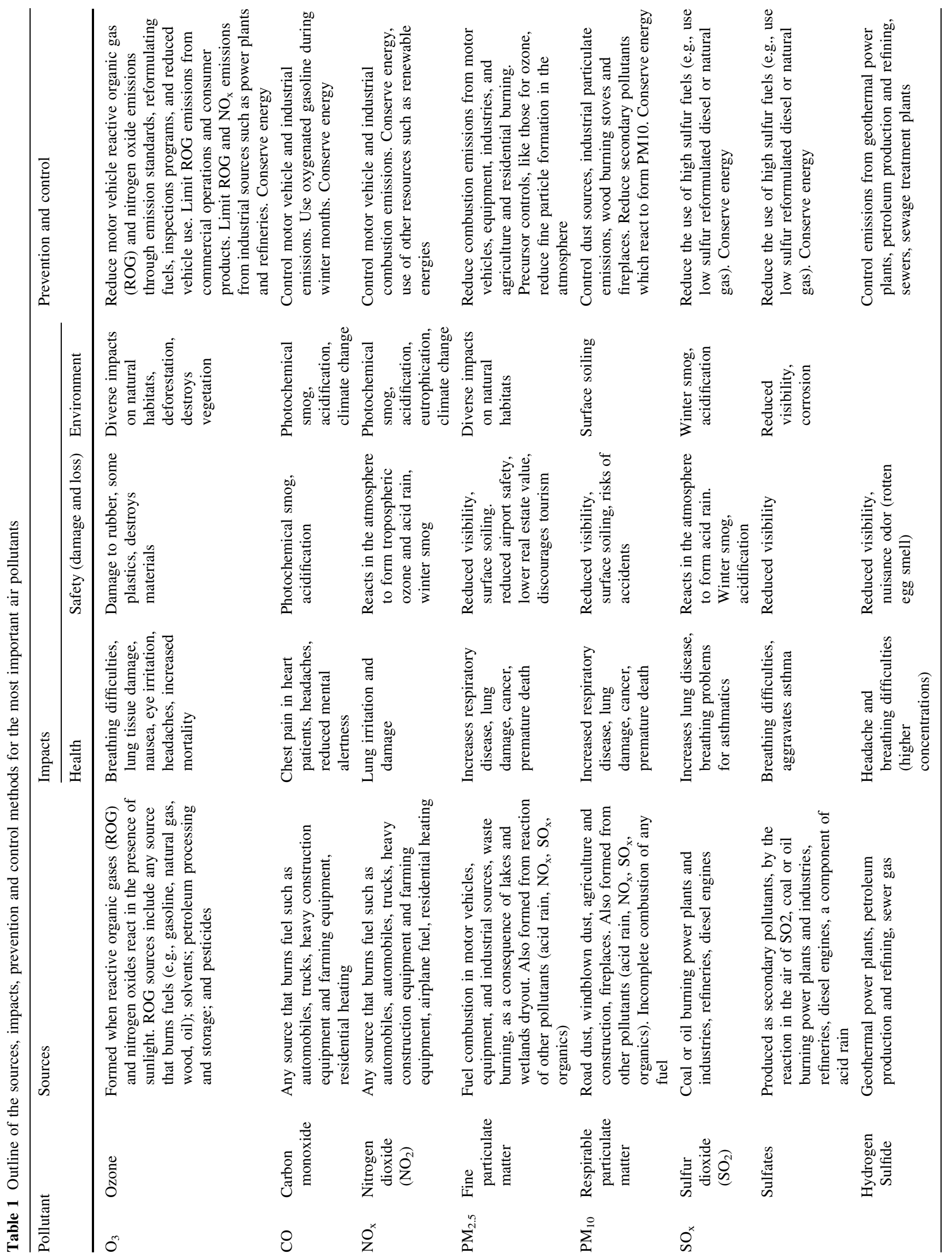


Table 2 The subjective severity of Air Quality Risk Index

\begin{tabular}{|c|c|c|c|c|}
\hline \multirow[t]{2}{*}{ Elements } & \multicolumn{4}{|c|}{ Subjective severity } \\
\hline & $\begin{array}{l}\text { Low } \\
1\end{array}$ & $\begin{array}{l}\text { Medium } \\
2\end{array}$ & $\begin{array}{l}\text { High } \\
3\end{array}$ & $\begin{array}{l}\text { Very high } \\
4\end{array}$ \\
\hline$R_{\mathrm{H}}(\mathrm{AQHI})$ & $1-3$ & $4-6$ & $7-10$ & More than 10 \\
\hline$R_{\mathrm{S}}$ [safety (damages and losses) (dollars per capita/per day)] & 0.00 & $0.00<L \leq 1.00$ & $1.00<L \leq 10.00$ & $\geq 10.00$ \\
\hline$R_{\mathrm{E}}(\mathrm{AQI})$ & $0-50$ & $51-100$ & $101-200$ & $201-500$ \\
\hline
\end{tabular}

Table 3 Air Quality Risk Index matrix

\begin{tabular}{|c|c|c|c|c|c|c|c|}
\hline \multirow{3}{*}{$\begin{array}{l}\text { Health } \\
R_{\mathrm{H}}\end{array}$} & \multirow{3}{*}{$\begin{array}{l}\text { Safety (damages } \\
\text { and losses) } \\
R_{\mathrm{S}}\end{array}$} & \multirow{3}{*}{$\begin{array}{l}\text { Environment } \\
R_{\mathrm{E}}\end{array}$} & \multirow{3}{*}{$\begin{array}{l}\text { Likelihood } \\
\text { of } \\
\text { occurrence }\end{array}$} & \multicolumn{4}{|c|}{ Subjective severity } \\
\hline & & & & Low & Medium & High & Very \\
\hline & & & & 1 & 2 & 3 & 4 \\
\hline Low undesirable health effect & Low loss & Low-destructive environmental effect & 1 & 1 & 2 & 3 & 4 \\
\hline Medium undesirable health effect & Medium loss & Medium-destructive environmental effect & 2 & 2 & 4 & 6 & 8 \\
\hline High undesirable health effect & High loss & High-destructive environmental effect & 3 & 3 & 6 & 9 & 12 \\
\hline $\begin{array}{l}\text { Very high undesirable health } \\
\text { effect }\end{array}$ & Very high loss & $\begin{array}{l}\text { Very high-destructive environmental } \\
\text { effect }\end{array}$ & 4 & 4 & 8 & 12 & 16 \\
\hline
\end{tabular}

Table 4 Air Quality Risk Index guideline

\begin{tabular}{|c|c|c|c|c|c|}
\hline \multirow[t]{2}{*}{ AQRI } & \multirow[t]{2}{*}{ Status } & \multirow[t]{2}{*}{ Color } & \multicolumn{3}{|l|}{ AQRI Guidline } \\
\hline & & & Health & Safety (damages and losses) & Environment \\
\hline $1-4$ & Good & Green & Suitable for outdoor activities & Business as usual & No measures needed \\
\hline $5-8$ & Medium & Yellow & $\begin{array}{l}\text { Perform usual activities, except in the } \\
\text { case vulnerable to air pollutants }\end{array}$ & $\begin{array}{l}\text { Setting some safety } \\
\text { measures }\end{array}$ & $\begin{array}{l}\text { Reducing the amount of pollutants } \\
\text { production }\end{array}$ \\
\hline $9-12$ & Critical & Red & Reduce outdoor to home activities & $\begin{array}{l}\text { Executing required } \\
\text { remediation plans }\end{array}$ & $\begin{array}{l}\text { Eliminating the related source of } \\
\text { pollutants }\end{array}$ \\
\hline $13-16$ & Catastrophic & Purple & Avoid outdoor activities & $\begin{array}{l}\text { Stop related activities until } \\
\text { the elimination of cause }\end{array}$ & $\begin{array}{l}\text { Reducing or changing the procedures } \\
\text { to eliminate related activities }\end{array}$ \\
\hline
\end{tabular}

Risk can be calculated from Table 3, by multiplying topical severity of each case to its likelihood of occurrence, Eq. (3).

The AQRI can be calculated from Eq. (4):

$\mathrm{AQRI}=\frac{R_{\mathrm{H}}+R_{\mathrm{S}}+R_{\mathrm{E}}}{3}$

$A Q R I$, Air Quality Risk Index; $R_{\mathrm{H}}$, health risk; $R_{\mathrm{S}}$, safety risk; $R_{\mathrm{E}}$, environment risk.

The evaluated amount of AQRI, as shown in Table 4, will provide a guideline which indicates the level of activities in order to safeguard the environment, health standards and safety measures to reduce the consequences risk (Abbaspour and Ahmadi 2014).

It is also possible to give weighting coefficient to each element. This depends on their importance as presented in Eq. (5):
$\mathrm{AQRI}_{\mathrm{W}}=\frac{R_{\mathrm{H}} W_{\mathrm{H}}+R_{\mathrm{S}} W_{\mathrm{S}}+R_{\mathrm{E}} W_{\mathrm{E}}}{W_{\mathrm{H}}+W_{\mathrm{S}}+W_{\mathrm{E}}}$

$R_{\mathrm{H}}$, health risk; $R_{\mathrm{S}}$, safety risk; $R_{\mathrm{E}}$, environment risk; $W$, weighted factors $0<w \leq 1$.

\section{Case study}

Tehran, Iran's capital and largest city, has the highest rate of urbanization with a population of 13.7 million in 2010 (Statistic Center of Iran 2011; The world bank 2013). This megacity has a large, densely populated area of $750 \mathrm{~km}^{2}$ (Sodoudi et al. 2014). It is located in valleys and is surrounded to the north, northwest, east and southeast by mountains with heights between 1000 and $3800 \mathrm{~m}$ (Madanipour 2006). 
Fig. 1 Levels of air pollutants in Tehran (in different years) (Ahadi et al. 2013)

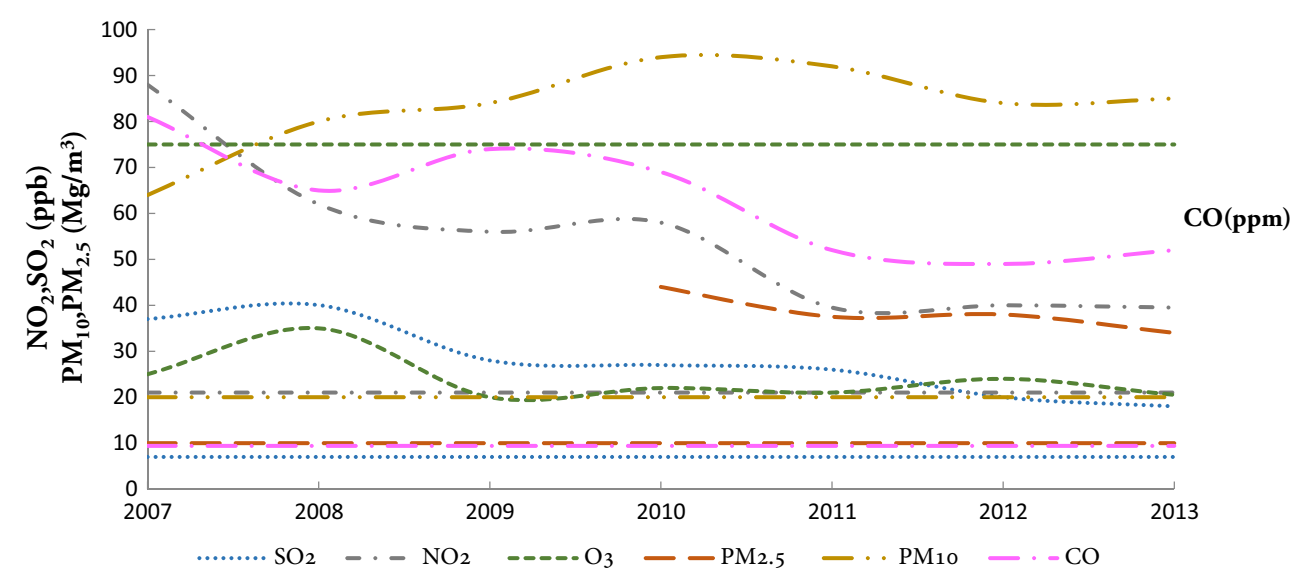

Fig. 2 The AQI for Tehran (in different years) (Ahadi et al. 2013)

Similar to other megacities of the world, Tehran faces serious air quality problems which are not only caused by its in habitants, but also caused by its geographical location (Naddafi et al. 2012).

The levels of air pollutants in Tehran throughout different years are shown in Fig. 1, and the AQI of Tehran for several years is shown in Fig. 2 (Ahadi et al. 2013).

In order to have a reliable estimation, the 2010 data were used to evaluate the state of AQRI. According to Air Quality Control Company (AQCC) report, levels of air pollutants in Fig. 1 are used.

Therefore, the AQHI $\left(\mathrm{SS}_{\mathrm{H}}\right)$ obtained from Eq. (1) is in the range of $1-3$, which means "low" see Table 2 .

Economic losses $\left(\mathrm{SS}_{\mathrm{S}}\right)$ due to air pollution according to the World Bank report in 2010 reached 10 milliard dollars, which, in terms of dollars per capita/per day, lies in "very high" zone level in Table 2.

Also AQI $\left(\mathrm{SS}_{\mathrm{E}}\right)$ has been reported by $\mathrm{AQCC}$ in the range of 51-100 for most of 2010 days which lies in "medium" range in Table 2.

As Fig. 2 illustrates, the likelihood of occurrence (LO) of unhealthy days is 104 , that is $30 \%$ of the year 2010, and stands as "medium" see Table 3.

Thus, by multiplying each subjective severity (SS) with its likelihood of occurrence (LO), the $R_{i}$ number will be specified and, by placing the $R_{i} \mathrm{~s}$ in AQRI formula, the $\mathrm{AQRI}$ therefore can be determined by the calculation of mean value of these three risk values.

The likelihood of occurrence for each subject can be determined according to the weighting coefficient and 
frequency of occurrence as defined. From Fig. 2, it can be illustrated that there are more than 104 days as unhealthy and, in 2014, only 20 days has been reported as healthy and clean, and from expert opinions, the related LOs, $R_{\mathrm{H}, \mathrm{S}, \mathrm{E}}$ are, respectively, 2, 3, 2:

$R_{\mathrm{H}}: 2 \times 2=4$

$R_{\mathrm{S}}: 4 \times 3=12$

$R_{\mathrm{E}}: 2 \times 2=4$

$\mathrm{AQRI} \rightarrow \frac{4+12+4}{3}=\frac{20}{3}=6.6$

Using Table 4, this indicates that the median range measure is required for this special case.

Even though AQI of the year 2010 for Tehran stands at clean zone, nevertheless, AQRI indicates that there is a need to change the common activities, establish safety measures and set new policies to reduce the amount of pollutants produced.

\section{Results and discussion}

The present study indicates the need for introducing a new AQI, which can cover all three factors of health, safety and environment with their related risks in an integrated manner.

AQRI is a steppingstone for decreasing financial losses and minimizing the environmental and related risks involved in achieving the sustainability and resiliency in cities.

The problem of AQI is that usually, the highest Ip (AQI of each pollutant) cannot reflect the actual situation of air quality.

The advantage of AQHI over AQI is that AQHI considers the integrated value of the three pollutants $\left(\mathrm{NO}_{2}\right.$, $\mathrm{PM}_{2.5}$ and $\mathrm{O}_{3}$ ), but its defect is that it does not include $\mathrm{CO}$ concentration.

As is clearly shown, AQI and AQHI cannot reflect the consequences of air pollution for megacities like Tehran, while AQRI can provide enough information for decision makers to take preventive or recovery measures and set required legislations.

\section{Conclusion}

This paper provides a new procedure to quantify the new index called AQRI. This index can be used as a tool for integrated urban air quality monitoring and can also be applied as a guideline for residential, commercial and industrial activities.

Since the risks of damage due to poor quality of air is evaluated in this index, one can provide a proper tool to take required measures which can help the resiliency of megacities such as Tehran.

The authors are currently working to improve this index by introducing some new parameters, such as weighting factors according to experts' opinions on each of the three factors of health, safety and environment.

Acknowledgments The authors sincerely thank Science and Research Branch of Islamic Azad University (SRBIAU) and the Center for Environment and Energy Research and Study (CEERS) of Science and Research Branch of Islamic Azad University for their support and also Air Quality Control Company (AQCC).

\section{References}

Abbaspour M, Ahmadi A (2014) Introduction of air quality risk index (AQRI) as a resilient urban air indicator. In: S.U.T. Symposium on Resilient Cities, Tehran

Ahadi S, Almasi S, Roshani M (2013) Tehran annual air quality report. s.n., Tehran

Chen H, Copes R (2013) Review of Air Quality Index and Air Quality Health Index. Queen's Printer for Ontario, Toronto

Committee on Environmental Health (2004) Ambient air pollution: health hazards to children. Pediatrics 114(6):1699-1707

Development Status Report of the Commissioner of the Environment (2009) Air Quality Health Index-Health Canada and Environment Canada. [Online]. http://www.oag-bvg.gc.ca/internet/ english/parl_cesd_200903_02_e

Environmental Protection Agency (2009) Air Quality Index. A guide to your health. [Online]. www.epa.gov/airnow. Accessed 2013

Gurjar B, Nagpure AS, Singh TP (2012) Air quality in megacities. [Online]. http://www.eoearth.org/view/article/149934

Hasselback P (2010) Air Quality Health Index variation across British Columbia. [Online]. www.bcairquality.ca/reports/pdfs/aqhivariation-bc.pdf?

Health Canada and Environment (2009) Status Report of the Commissioner of the Environment and Sustainable Development. Air Quality Health Index, Canada

Health Protection Agency for the Committee on the Medical Effects of Air Pollutants (2011) Review of the UK Air Quality Index. s.n, UK

Hidy G, Pennell W (2010) Multipollutant Air Quality Management. Air and Waste Management Association

House of Commons Environmental Audit Committee (2010) Air quality fifth report of session 2009-10. Authority of the House of Commons, London

Kansas Department of Health and Environment Bureau (2005) Kansas Air Quality Report. [Online]. http://www.kdheks.gov/ bar/download/2004-2005_AQ_Report.pdf

Kassomenos P, Papaloukas C, Petrakis M, Karakitsios S (2008) Assessment and prediction of short term hospital admissions: the case of Athens, Greece. Atmos Environ 42:7078-7086

Katsouyanni K et al (1996) Short term effects of air pollution on health: a European approach using epidemiologic time series data: the APHEA protocol. Epidemiol Community Health 50:S12-S18

Madanipour A (2006) Urban planning and development in Tehran. Cities 23(6):433-438

Naddafi K, Hassanvand MS, Yunesian M, Momeniha F, Nabizadeh R, Faridi S, Gholampour A (2012) Health impact assessment of air pollution in megacity of Tehran, Iran. Iran J Environ Health Sci Eng 9:28 
Ontario Ministry of the Environment (2005) Oriento, protecting our environment green facts Ontario's Air Quality Index. [Online]. www.ene.gov.on.ca/stdprodconsume/groups/lr/.../std01_079055. pdf. Accessed 2013

Ontario Ministry of the Environment (2006) Environmental monitoring and reporting branch. [Online]. www.ene.gov.on.ca/ stdprodconsume/groups/lr/.../std01_079055.pdf

Prinn RG et al (2005) Science and policy of global change. MIT Joint Program on the Science and Policy of Global Change, Cambridge

Sicard P et al (2012) The Aggregate Risk Index: an intuitive tool providing the health risks of air pollution to health care community and public. Atmos Environ 46:11-16

Sodoudi S et al. (2014) Mitigating the urban heat Island effect in Megacity Tehran. Hindawi Publishing Corporation Advances in Meteorology

Statistic Center of Iran (2011) SCI: Statistic Center of Iran. [Online]. www.amar.org.ir

Sunyera J et al (2003) The association of daily sulfur dioxide air pollution levels with hospital admissions for cardiovascular diseases in Europe (The Aphea-II study). Eur Heart J 24:752-760

The World Bank (2013) Population. [Online]. http://data.worldbank. org/indicator/SP.POP.TOTL. Accessed 2013

U.S. Environmental Protection Agency (1997) The benefits and costs of the clean Air Act, 1970 to 1990. s.n, s.l.

U.S. Environmental Protection Agency (2006) Guidelines for the reporting of daily air quality - the Air Quality Index (AQI). s.n, North Carolina

U.S. Environmental Protection Agency of Standards (2006) Guidelines for the reporting of daily air quality - the Air Quality Index (AQI). [Online]. www.epa.gov/ttn/caaa/t1/memoranda/rg701. pdf?

World Health Organization (2010) Exposure to air pollution: a major public health concern. WHO Document Production Services, Geneva

World Health Organization (2014) Ambient (outdoor) air quality and health. [Online]. WHO Fact Sheet No. 313; http://www.who.int/ mediacentre/factsheets/fs313/en/index.html. Accessed 2014 